\title{
GLOBAL SOLUTIONS OF THE RANDOM VORTEX FILAMENT EQUATION
}

\author{
Z. BRZEŹNIAK ${ }^{\dagger}$, M. GUBINELLI ${ }^{\ddagger}$ AND M. NEKLYUDOV $^{\dagger}$
}

\begin{abstract}
We prove the existence of a global solution for the filament equation with inital condition given by a geometric rough path in the sense of Lyons [17]. Our work gives a positive answer to a question left open in recent publications: Berselli and Gubinelli [5] showed the existence of a global solution for a smooth initial condition while Bessaih, Gubinelli, Russo [6] proved the existence of a local solution for a general initial condition given by a rough path.
\end{abstract}

\section{THE FILAMENT EQUATION}

In this note we prove the existence of a global solution for the following rough filament equation

$$
\left\{\begin{array}{l}
\frac{d \gamma(t)(\xi)}{d t}=u^{\gamma(t)}(\gamma(t)(\xi)), \quad t \in[0, \infty), \xi \in[0,1], \\
\gamma(0)=\gamma_{0},
\end{array}\right.
$$

where the initial condition $\gamma_{0}:[0,1] \rightarrow \mathbb{R}^{3}$ is a geometric $\nu$-rough path (for some $\nu \in\left(\frac{1}{3}, 1\right)$ ), see Assumption 2.6. Here $\gamma:[0, \infty) \rightarrow \mathcal{D}_{\gamma_{0}} \subset \mathcal{C}$ is some trajectory in the subset $\mathcal{D}_{\gamma_{0}}$ of the space $\mathcal{C} \subseteq C\left([0,1] ; \mathbb{R}^{3}\right)$ of continuous closed curves in $\mathbb{R}^{3}$ parametrized by the interval $[0,1]$. Finally $u^{\gamma}, \gamma \in \mathcal{D}_{\gamma_{0}}$ is a vector field given by

$$
u^{\gamma}(x)=\int_{0}^{1} \nabla \phi(x-\gamma(\xi)) \times d \gamma(\xi),
$$

where $\phi: \mathbb{R}^{3} \rightarrow \mathbb{R}$ is a smooth function which satisfies certain assumptions (see Hypothesis 3.2). The exact meaning of the line integral above and the definition of the set $\mathcal{D}_{\gamma_{0}}$ will be given below. Equation (1.1) has its origin in the theory of the three dimensional Euler equations. It is well known that for the two dimensional Euler equations the vorticity $\omega=\operatorname{curl} u$ is transported along the fluid flow. The situation changes drastically in three dimensional case. Additional "stretching" term in the equation defining the vorticity leads to a possibility of its blow up in finite time. Furthermore, a result of Beale, Kato, and Majda [2] suggests that a possible singularity of the Euler equations appears when the vorticity field of the fluid blows up. Consequently, understanding the behaviour of the vorticity of an ideal incompressible fluid is an important problem in fluid dynamics.

The properties of the motion of the vorticity has been studied for the last 150 years starting from the works of Helmholtz [15] and Kelvin [16]. It has been suggested by Kelvin to use the so called Biot-Savart law

$$
u(x)=\int_{\mathbb{R}^{3}} \frac{x-y}{|x-y|^{3}} \times \omega(y) d y, \quad x \in \mathbb{R}^{3}
$$

Date: January 16, 2014.

This research has been supported by the ANR Project ECRU (ANR-09-BLAN-0114-01/2). 
where $\times$ denotes the vector product in $\mathbb{R}^{3}$, combined with an assumption that the vorticity is supported by a smooth closed curve evolving in time $\gamma: \mathbb{R}_{+} \times[0,1] \rightarrow \mathbb{R}^{3}$ via a formula

$$
\omega(x, t)=\Gamma \int_{0}^{1} \delta(x-\gamma(t, \xi)) \frac{\partial \gamma(t, \xi)}{\partial \xi} d \xi, \quad x \in \mathbb{R}^{3}, t \geq 0
$$

and definition of the flow

$$
\left\{\begin{aligned}
\frac{d X_{t}(x)}{d t} & =u\left(X_{t}(x), t\right), \quad t \geq 0 \\
X_{0}(x) & =x
\end{aligned}\right.
$$

to formally deduce the filament equation

$$
\frac{\partial \gamma}{\partial t}(t, \xi)=-\frac{\Gamma}{4 \pi} \int_{0}^{1} \frac{\gamma(t, \xi)-\gamma(t, \eta)}{|\gamma(t, \xi)-\gamma(t, \eta)|^{3}} \times \frac{\partial \gamma(t, \eta)}{\partial \eta} d \eta
$$

The assumption that the vorticity is supported by some curve is coherent with numerical simulations of 3D turbulent fluids which show that regions of large vorticity have a form of a "filament", see for instance [3] and [21].

Equation (1.4) has singularity when $\xi$ and $\eta$ are close to each other and the initial curve $\gamma$ is smooth. As a consequence, the energy of the solution of this equation given by the formula

$$
E(t)=\frac{\Gamma^{2}}{8 \pi} \int_{0}^{1} \int_{0}^{1} \frac{1}{|\gamma(t, \xi)-\gamma(t, \eta)|} \frac{\partial \gamma(t, \eta)}{\partial \eta} \cdot \frac{\partial \gamma(t, \xi)}{\partial \xi} d \xi d \eta, \quad t \geq 0
$$

is infinite for any smooth curve $\gamma(t, \cdot)$. In order to avoid the singularity Rosenhead [20] introduced the regularized model

$$
\frac{\partial \gamma}{\partial t}(t, \xi)=-\frac{\Gamma}{4 \pi} \int_{0}^{1} \frac{\gamma(t, \xi)-\gamma(t, \eta)}{\left(|\gamma(t, \xi)-\gamma(t, \eta)|^{2}+\mu^{2}\right)^{3 / 2}} \times \frac{\partial \gamma(t, \eta)}{\partial \eta} d \eta
$$

which is widely used in aereonautics to study the dynamics of trailing vortices at the tips of airplane wings.

The problem (1.1) has been studied by Berselli and Bessaih [4] and then by Berselli and Gubinelli [5]. It contains the equation (1.5) as a particular case when, for for some $\mu>0$,

$$
\phi(x)=\frac{\Gamma}{\left(|x|^{2}+\mu^{2}\right)^{\frac{1}{2}}}, \quad x \in \mathbb{R}^{3} .
$$

Equation (1.1) is in fact a nonlinear PDE for a function $\gamma:[0, \infty) \times[0,1] \rightarrow \mathbb{R}^{3}$. A natural setting for the well-posedness of the corresponding Cauchy problem is obtained by requiring the vector field $u$ to be well defined and Lipshitz with respect to the space variable. To this effect the approach followed in [4] is to set up the equation as an evolution problem in the Sobolev space $H^{1}$ of closed absolutely continuous curves in $\mathbb{R}^{3}$ with square integrable first derivative (with respect to the parameter). This approach implies that the vector field (once lifted to $H^{1}$ ) does not allow strong enough estimates to deduce the global existence. This issue is ultimately due to the fact that in the 3-dimensional incompressible flows, the vortices stretch and undergo a complex dynamics and that a priori this could lead to a explosion of the $H^{1}$ norm. Such a difficulty should be compared to the more stable behavior of $2 \mathrm{~d}$ vortex points which, under incompressible flows, are simply transported along the flow lines. Exploiting the conservation of the kinetic energy of the flow and a control of the velocity field generated by the vortex line via the associated kinetic energy Berselli and Gubinelli [5] showed the existence of a global solution to equation (1.1) with initial conditions in $H^{1}$. 
Bessaih, Gubinelli and Russo [6], partially motivated by the random filament models suggested by Gallavotti [11] and Chorin [8], considered the above evolution problem when the initial data is a random closed curve. For definiteness they considered the initial data sampled from the law of a 3-dimensional Brownian loop (a Brownian motion starting at $0 \in \mathbb{R}^{3}$ and conditioned to return to $0 \in \mathbb{R}^{3}$ at time 1 ). In this case it is no more possible to set up the problem in the Sobolev space $H^{1}$ since Brownian trajectories almost surely do not belong to this space. A more serious problem is the meaning to give to the generalized Biot-Savart relation (1.2) since the line integral along a Brownian trajectory does not allow a straighforward definition. Moreover stochastic integration (à la Itô or Stratonovich) does not provide a good framework to study this problem since it does not posses a natural filtration on the parameter space stable under the time evolution. For these reasons [6] identified the spaces of controlled rough paths as a natural framework to have a well posed problem.

Rough path theory has been introduced by T. J. Lyons in the seminal paper [17] (see also [10, $18,19])$ as a way to overcome certain difficulties of stochastic integration theories and have a robust analytical framework to solve stochastic differential equations and similar problems involving integration of non-regular vector-fields. It turns out that rough paths theory and in particular the notion of controlled paths introduced in [12] allows to give a natural interpretation to the Biot-Savart relation (1.2) and obtain a well-posed problem. Using this approach Bessaih, Gubinelli, Russo [6] obtained existence of a local solution to eq. (1.1) when the initial data is a closed curve of Hölder class with exponent $\nu \in(1 / 3,1]$ (suitably lifted to rough path space).

The aim of the present paper is to extend the energy method of Berselli and Gubinelli to the rough path setting and obtain a global existence result for the equation (1.1) when the initial data is a geometric rough path, thus completing the analysis of [6].

Recently there have been some deep progresses in the study of evolution equations in the space of controlled rough paths. In particular Hairer [13] showed how to use controlled path theory to have well-posedness of a multidimensional Burgers type equation driven by additive space-time white noise and later [14] used similar ideas to tackle the longstanding open problem of the Kardar-Parisi-Zhang equation for which he described a notion of solution for which existence and uniqueness can be proven.. The key technical tool to obtain these results has been the observation that the more singular non-linear term involved in the fixed-point argument has the same structure of the Biot-Savart relation (1.2) and thus can be similarly handled via controlled paths techniques.

A major open problem which remains largely unexplored is the rigorous study of the unregularized filament equation (1.4), whose singularity seems to defy any reasonable analytic approach. On this respect the very suggestive heuristic computations of Gallavotti [11] seems to hint to the fact that rapid oscillations of the curve $\gamma$ could provide a natural regularization. Another very interesting problem is the study of the rough filament equation as an Hamiltonian system on the space of parametrization invariant paths on the lines of [1] and [7].

Acknowledgments. The authors would like to thank the anonymous referees for their careful reading and the detailed comments which helped us to improve the presentation. 


\section{Definition and Properties of Rough Path integrals}

In this section we will present the controlled path framework and we will define the rough path integral and state some of its properties. We will mainly follow the papers [12] and [6].

In what follows by $V, W$ we will usually denote Banach spaces, by $L(V, W)$ the Banach space of bounded linear maps from $V$ to $W$ and by $C(X, Y)$ the space of continuous function from $X$ to $Y$. Moreover we let $\mathbb{T}=[0,1]$ and

$$
C_{n}(V)=\left\{f \in C\left(\mathbb{T}^{n}, V\right): f\left(t_{1}, t_{2}, \cdots, t_{n}\right)=0 \text { if } t_{i}=t_{i+1} \text { for some } i=1, . ., n-1\right\} .
$$

We will understand that $C(V)=C_{1}(V)$ and we will consider the Hölder norm

$$
|f|_{\mu}=\sup _{a \in \mathbb{T}}|f(a)|+\sup _{0 \leq a<b \leq 1} \frac{|f(a)-f(b)|_{V}}{|a-b|^{\mu}}, \quad f \in C_{1}(V) ;
$$

on $C_{1}(V)$ and the following semi-norms on the spaces $C_{2}(V)$ and $C_{3}(V)$

$$
|f|_{\mu}=\sup _{0 \leq a<b \leq 1} \frac{|f(a, b)|_{V}}{|a-b|^{\mu}} ; \quad f \in C_{2}(V), \quad|g|_{\mu}=\sup _{0 \leq a<b<c \leq 1} \frac{|g(a, b, c)|_{V}}{|a-c|^{\mu}}, g \in C_{3}(V) .
$$

The associated normed spaces are defined by

$$
\begin{gathered}
C^{\mu}(V)=C_{1}^{\mu}(V)=\left\{f \in C_{1}(V):|f|_{\mu}<\infty\right\}, \\
C_{2}^{\mu}(V)=\left\{f \in C_{2}(V):|f|_{\mu}<\infty\right\}, \quad C_{3}^{\mu}(V)=\left\{g \in C_{3}(V):|g|_{\mu}<\infty\right\} .
\end{gathered}
$$

For $n=1,2$ define the operators $\delta-\delta_{n}: C_{n}(V) \rightarrow C_{n+1}(V)$ as

$$
\delta_{1} f(a, b)=f(b)-f(a), \quad \delta_{2} g(a, b, c)=g(a, c)-g(a, b)-g(b, c)
$$

for all $a, b, c \in \mathbb{T}$. They satisfy the following fundamental property $\delta \delta f=0$, for all $f \in C_{1}(V)$. Let $\mathcal{Z} C_{2}^{\mu}(V)=C_{3}^{\mu}(V) \cap \operatorname{ker} \delta$ and $\mathcal{B} C_{2}^{\mu}(V)=C_{3}^{\mu}(V) \cap \operatorname{im} \delta$. If $g \in \mathcal{Z} C_{2}^{\mu}(V)$ then $g \in$ $\mathcal{B} C_{2}^{\mu}(V)$, i.e. is there exists $f \in C_{1}(V)$ such that $\delta f=g$. Then following result has been proved in [12]:

Proposition 2.1 (Sewing lemma). For any $\mu>1$ there exists an unique linear map (Sewing map) $\Lambda: \mathcal{Z} C_{3}^{\mu}(V) \rightarrow C_{2}^{\mu}(V)$ such that

$$
\delta \Lambda=\operatorname{id}_{\mathcal{Z} C_{3}^{\mu}(V)} .
$$

This map is continuous and we have

$$
\|\Lambda h\|_{\mu} \leq \frac{1}{2^{\mu}-2}\|h\|_{\mu}, \quad h \in \mathcal{Z} C_{3}^{\mu}(V) .
$$

Now, we define a class of paths for which rough path integral will be defined.

Definition 2.2. Assume that $\nu \in(0,1)$ and fix $X \in C^{\nu}(V)$. We say that a path $Y \in C(V)$ is weakly controlled by $X$ if there exist a function $Y^{\prime} \in C^{\nu}(L(V, V))$ such that the function

$$
R(\xi, \eta)=Y(\xi)-Y(\eta)-Y^{\prime}(\eta)(X(\xi)-X(\eta)), \quad \xi, \eta \in \mathbb{T},
$$

belongs to $C_{2}^{2 \nu}(V)$. We denote $\mathcal{D}_{X}$ the set of all the pairs $\left(Y, Y^{\prime}\right)$ satisfying (2.1). This is a vector space which can be endowed with the semi-norm $\|\cdot\|_{\mathcal{D}_{X}}$ given by the following formula

$$
\left\|\left(Y, Y^{\prime}\right)\right\|_{\mathcal{D}_{X}}=\left|Y^{\prime}\right|_{C^{\nu}}+\|R\|_{C_{2}^{2 \nu}},
$$


Furthermore, let us define a norm $\|\cdot\|_{\mathcal{D}_{X}}^{*}$ in $\mathcal{D}_{X}$ by

$$
\left\|\left(Y, Y^{\prime}\right)\right\|_{\mathcal{D}_{X}}^{*}=\|Y\|_{\mathcal{D}_{X}}+\sup _{\xi \in \mathbb{T}}|Y(\xi)| .
$$

With these definitions $\left(\mathcal{D}_{X},\|\cdot\|_{\mathcal{D}_{X}}^{*}\right)$ is a Banach space. While not necessary for the integration theory it will convenient for our intended application to assume that all our controlled paths take values in $V=\mathbb{R}^{3}$ and are closed, that is $Y(0)=Y(1)$.

In the present paper an element $\left(Y, Y^{\prime}\right) \in \mathcal{D}_{X}$ can always be canonically identified from its first component $Y$ by the mean of standard procedures discussed below. In this context we denote by $R^{Y}$ the corresponding remainder: $R^{Y}=\delta Y-Y^{\prime} \delta X$. Moreover we will often omit to specify $Y^{\prime}$ when it is clear from the context and write $\|Y\|_{\mathcal{D}_{X}}$ instead of $\left\|\left(Y, Y^{\prime}\right)\right\|_{\mathcal{D}_{X}}$.

We will repeatedly use the basic estimate

$$
\|Y\|_{C^{\nu}} \leq\|Y\|_{\mathcal{D}_{X}}^{*}\left(1+\|X\|_{C^{\nu}}\right) .
$$

Controlled paths are stable by mapping via sufficiently regular functions.

Lemma 2.3. Let $\phi \in C^{2}\left(\mathbb{R}^{3}, \mathbb{R}^{3}\right)$ and $\left(Y, Y^{\prime}\right) \in \mathcal{D}_{X}$. Then

$$
\left(W, W^{\prime}\right):=\left(\phi(Y), \phi^{\prime}(Y) Y^{\prime}\right) \in \mathcal{D}_{X}
$$

and the corresponding remainder has the following representation

$$
\begin{aligned}
R^{W}(\xi, \eta)= & \phi^{\prime}(Y(\xi)) R^{Y}(\xi, \eta)+(Y(\eta)-Y(\xi)) \times \\
& \times \int_{0}^{1}[\nabla \phi(Y(\xi)+r(Y(\eta)-Y(\xi)))-\nabla \phi(Y(\xi))] d r, \quad \xi, \eta \in \mathbb{T} .
\end{aligned}
$$

Furthermore, there exists a constant $K \geq 1$ such that

$$
\|\phi(Y)\|_{\mathcal{D}_{X}} \leq K\|\nabla \phi\|_{C^{1}}\|Y\|_{\mathcal{D}_{X}}\left(1+\|Y\|_{\mathcal{D}_{X}}\right)\left(1+\|X\|_{\nu}\right)^{2} .
$$

Moreover, if $\left(\tilde{Y}, \tilde{Y}^{\prime}\right) \in \mathcal{D}_{\tilde{X}}$ and $\left(\tilde{W}, \tilde{W}^{\prime}\right):=\left(\phi(\tilde{Y}), \phi^{\prime}(\tilde{Y}) \tilde{Y}^{\prime}\right)$ then

$$
\begin{array}{r}
\left\|W^{\prime}-\tilde{W}^{\prime}\right\|_{\nu}+\left\|R^{W}-R^{\tilde{W}}\right\|_{2 \nu}+\|W-\tilde{W}\|_{\nu} \leq \\
C\left(\|X-\tilde{X}\|_{\nu}+\left\|Y^{\prime}-\tilde{Y}^{\prime}\right\|_{\nu}+\left\|R^{Y}-R^{\tilde{Y}}\right\|_{2 \nu}+\|Y-\tilde{Y}\|_{\nu}\right)
\end{array}
$$

with

$$
C=K\|\phi\|_{C^{3}}\left(1+\|X\|_{C^{\nu}}+\|\tilde{X}\|_{C^{\nu}}\right)^{3} \mid\left(1+\|Y\|_{\mathcal{D}_{X}}+\|\tilde{Y}\|_{\mathcal{D}_{\tilde{X}}}\right)^{2} .
$$

When $X=\tilde{X}$ we have

$$
\begin{aligned}
&\|\phi(Y)-\phi(\tilde{Y})\|_{\mathcal{D}_{X}} \leq K\|\nabla \phi\|_{C^{2}}\|Y\|_{\mathcal{D}_{X}} \\
&\left(1+\|Y\|_{\mathcal{D}_{X}}+\|\tilde{Y}\|_{\mathcal{D}_{X}}\right)^{2}\left(1+\|X\|_{C^{\nu}}\right)^{4}\|Y-\tilde{Y}\|_{\mathcal{D}_{X}} .
\end{aligned}
$$

Proof. See [12], Proposition 4 for all statements of the Lemma, except (2.6) (which is actually also proven, though not stated explicitly). Let us show (2.6). Denote $y(r)=Y(\xi)+r(Y(\eta)-$ 
$Y(\xi)), r \in[0,1]$. Then

$$
\begin{aligned}
& \phi(y(1))-\phi(y(0))=\int_{0}^{1} \phi^{\prime}(y(r)) y^{\prime}(r) d r \\
= & \sum_{k}\left(Y^{k}(\eta)-Y^{k}(\xi)\right) \int_{0}^{1} \frac{\partial \phi}{\partial x_{k}}(y(r)) d r=\sum_{k} \frac{\partial \phi}{\partial x_{k}}(Y(\xi))\left(Y^{k}(\eta)-Y^{k}(\xi)\right) \\
+ & \sum_{k}\left(Y^{k}(\eta)-Y^{k}(\xi)\right) \int_{0}^{1}\left[\frac{\partial \phi}{\partial x_{k}}(y(r))-\frac{\partial \phi}{\partial x_{k}}(Y(\xi))\right] d r \\
= & \sum_{k, l} \frac{\partial \phi}{\partial x_{k}}(Y(\xi))\left(Y^{\prime}\right)^{k l}\left(X^{l}(\eta)-X^{l}(\xi)\right)+\sum_{k} \frac{\partial \phi}{\partial x_{k}}(Y(\xi))\left(R^{Y}\right)^{k}(\xi, \eta) \\
+ & \sum_{k}\left(Y^{k}(\eta)-Y^{k}(\xi)\right) \int_{0}^{1}\left[\frac{\partial \phi}{\partial x_{k}}(y(r))-\frac{\partial \phi}{\partial x_{k}}(Y(\xi))\right] d r
\end{aligned}
$$

and the result follows.

In order to define the integral for paths controlled by $X$ we need a further ingredient: the existence of basic integrals of $X$ with respect to $X$ itself. These objects are encoded into what is refereed to as a rough path as follows.

Definition 2.4. Assume that $\nu>1 / 3$. We say that couple $\mathbb{X}=\left(X, \mathbb{X}^{2}\right)$ where $X \in C^{\nu}\left(\mathbb{T}, \mathbb{R}^{3}\right)$ and $\mathbb{X}^{2} \in C_{2}^{2 \nu}\left(L\left(\mathbb{R}^{3}, \mathbb{R}^{3}\right)\right)$ is a $\nu$-rough path if the following condition is satisfied:

$$
\mathbb{X}^{2}(\xi, \rho)-\mathbb{X}^{2}(\xi, \eta)-\mathbb{X}^{2}(\eta, \rho)=(X(\xi)-X(\eta)) \otimes(X(\eta)-X(\rho)), \quad \xi, \eta, \rho \in \mathbb{T} .
$$

Remark 2.5. If $\nu>1 / 2$ the solution $\mathbb{X}^{2}$ to (2.12) is unique. Indeed, assume that there exists another $\mathbb{X}_{1}^{2}$ which satisfies definition 2.4. Put $G(\xi)=\mathbb{X}^{2}(\xi, 0)-\mathbb{X}_{1}^{2}(\xi, 0)$. Then by condition (2.12)

$$
\mathbb{X}^{2}(\xi, \rho)-\mathbb{X}_{1}^{2}(\xi, \rho)=G(\xi)-G(\rho),
$$

and, since $\mathbb{X}^{2} \in C_{2}^{2 \nu}, G$ is a Hölder function of order bigger than 1. Hence, $G=0$ and thus $\mathbb{X}_{1}^{2}=\mathbb{X}^{2}$

If $\nu>1$ and $\mathbb{X}$ is a $\nu$-rough path, then $\mathbb{X}$ is given by the pair of constants $(X(0), 0)$. Indeed, in this case $X$ is Hölder function with exponent more than 1 i.e. $X$ is equal to a constant function $X(0)$ and the only possible choice for the second component is $\mathbb{X}^{2}=0$.

If $\nu \in(1 / 2,1]$ then $\mathbb{X}^{2}$ is uniquely determined by $X$. Indeed, if we let

$$
\mathbb{X}^{2, i j}(\xi, \eta)=\int_{\xi}^{\eta}\left(X_{\rho}^{i}-X_{\eta}^{i}\right) d X_{\rho}^{j}, \xi, \eta \in \mathbb{T} i, j=1,2,3,
$$

where the integral is understood in the sense of Young (see [22]) it is possible to show that $\mathbb{X}^{2} \in C_{2}^{2 \nu}$ and that this definition satisfies conditions of Definition 2.4. Since $\nu>1 / 2$ it is the only possible choice. 
Definition 2.6. We say that our $\nu$-rough path $\left(X, \mathbb{X}^{2}\right)$ is a geometric $\nu$-rough path if there exist a sequence $\left(X_{n}, \mathbb{X}_{n}^{2}\right)$ such that $X_{n} \in C\left(\mathbb{T}, \mathbb{R}^{3}\right)$ is piecewise smooth, and

$$
\lim _{n \rightarrow \infty}\left[\left\|X_{n}-X\right\|_{\nu}+\left\|\mathbb{X}_{n}^{2}-\mathbb{X}^{2}\right\|_{2 \nu}\right]=0
$$

where

$$
\mathbb{X}_{n}^{2, i j}(\xi, \eta)=\int_{\xi}^{\eta}\left(X_{n}^{i}(\rho)-X_{n}^{i}(\eta)\right) d X_{n}^{j}(\rho), \quad \xi, \eta \in \mathbb{T}, i, j=1,2,3 .
$$

Example 2.7. Let $\left\{B_{t}\right\}_{t \in[0,1]}$ be the standard 3-dimensional Brownian bridge such that $B_{0}=$ $B_{1}=x_{0}$ and let $\mathbb{B}^{2, i j}, i, j=1,2,3$, be the area processes defined by

$$
\mathbb{B}^{2, i j}(\xi, \eta)=\int_{\xi}^{\eta}\left(B_{\rho}^{i}-B_{\eta}^{i}\right) d B_{\rho}^{j}
$$

where the integral can be understood either in the Stratonovich or in the Itô sense. Then, the couple $\left(B, \mathbb{B}^{2}\right)$ is a $\nu$-rough path for all $\nu \in(1 / 3,1 / 2)$ (see [6, p.1849]). Moreover, if the integral is understood in the Stratonovich sense, then $\left(B, \mathbb{B}^{2}\right)$ is also a geometric $\nu$-rough path. Indeed, it follows from Theorem 3.1 in [9] that one can approximate $X$ with piecewise linear dyadic $\left(X_{n}\right)_{n \geq 1}$ in the sense of Definition 2.6 where the limit is understood in the almost sure sense.

From now on we suppose that the geometric $\nu$-rough path $\mathbb{X}=\left(X, \mathbb{X}^{2}\right)$ with $\nu>1 / 3$ and the corresponding Banach space $\mathcal{D}_{X}$ are fixed. For a finite partition $\pi=\left\{\xi_{0}=\xi<\xi_{1}<\cdots<\right.$ $\left.\xi_{n}=\eta\right\}$ be of the interval $[\xi, \eta]$, let $d(\pi)=\sup _{i}\left|\xi_{i+1}-\xi_{i}\right|$ denote the mesh size of the partition $\pi$.

Lemma 2.8. If $Y, Z \in \mathcal{D}_{X}$ then the limit

$$
\lim _{d(\pi) \rightarrow 0} \sum_{i=0}^{n-1}\left[Y\left(\xi_{i}\right)\left(Z\left(\xi_{i+1}\right)-Z\left(\xi_{i}\right)\right)+Y^{\prime}\left(\xi_{i}\right) Z^{\prime}\left(\xi_{i}\right) \mathbb{X}^{2}\left(\xi_{i+1}, \xi_{i}\right)\right]=: \int_{\xi}^{\eta} Y d Z
$$

exists and defined the integral on the r.h.s.

Proof. This statement is a direct consequence of the existence and properties of the sewing map and of the definition of the controlled path space, see [12], Theorem 1. For the sake of the presentation we sketch a proof. Let us denote

$$
A\left(\xi^{\prime}, \eta^{\prime}\right)=Y\left(\xi^{\prime}\right)\left(Z\left(\eta^{\prime}\right)-Z\left(\xi^{\prime}\right)\right)+Y^{\prime}\left(\xi^{\prime}\right) Z^{\prime}\left(\xi^{\prime}\right) \mathbb{X}^{2}\left(\eta^{\prime}, \xi^{\prime}\right), \quad \eta^{\prime}, \xi^{\prime} \in \mathbf{T} .
$$

Let us note that, with the assumptions of the Lemma, $\delta A \in C_{3}^{3 \nu}$. Hence, provided that $3 \nu>1$, we can apply the sewing map and we have the decomposition $A=\delta I+\Lambda \delta A$ where $I \in C_{1}$. But this implies that

$$
\lim _{d(\pi) \rightarrow 0} \sum_{i=0}^{n-1} A\left(\xi_{i}, \xi_{i+1}\right)=\lim _{d(\pi) \rightarrow 0} \sum_{i=0}^{n-1}\left(I\left(\xi_{i+1}\right)-I\left(\xi_{i}\right)\right)+\lim _{d(\pi) \rightarrow 0} \sum_{i=0}^{n-1}\left(\Lambda \delta A\left(\xi_{i}, \xi_{i+1}\right)\right) .
$$


The first term on the r.h.s. telescopes and since $\left|\Lambda \delta A\left(\xi_{i}, \xi_{i+1}\right)\right| \lesssim\left|\xi_{i+1}-\xi_{i}\right|^{3 \nu}$ the second term on the r.h.s converges to zero proving the existence of the limit and moreover

$$
\lim _{d(\pi) \rightarrow 0} \sum_{i=0}^{n-1} A\left(\xi_{i}, \xi_{i+1}\right)=I(\eta)-I(\xi) .
$$

The explicit representation in terms of the sewing map can be used to prove the various estimates for the rough integral below.

Remark 2.9. In the case of $\nu>1 / 2$ the line integral defined in Lemma 2.8 is reduced to the Young definition of the line integral $\int Y d Z$. Indeed, it is enough to notice that second term in formula (2.15) is of the order $O\left(\left|\xi_{i+1}-\xi_{i}\right|^{2 \nu}\right), 2 \nu>1$. In this case the line integral does not depend upon $Y^{\prime}, Z^{\prime}$.

Integrals of controlled paths have very nice continuity property with respect to variations of the controlled path and also with respect variations of the rough path on which the controlled path space is modelled.

In the following $\mathbb{X}=\left(X, \mathbb{X}^{2}\right)$ and $\tilde{\mathbb{X}}=\left(\tilde{X}, \tilde{\mathbb{X}}^{2}\right)$ are two $\nu$-rough paths and $Y \in \mathcal{D}_{X}$ and $\tilde{Y} \in \mathcal{D}_{\tilde{X}}$ two path respectively controlled by $X$ and $\tilde{X}$. In this case we introduce the following distance

$$
D(Y, \tilde{Y})=\|X-\tilde{X}\|_{C^{\nu}}+\left\|\mathbb{X}^{2}-\tilde{\mathbb{X}}^{2}\right\|_{C_{2}^{2 \nu}}+\left\|Y^{\prime}-\tilde{Y}^{\prime}\right\|_{C^{\nu}}+\left\|R^{Y}-R^{\tilde{Y}}\right\|_{C_{2}^{2 \nu}}+\|Y-\tilde{Y}\|_{C^{\nu}} .
$$

Lemma 2.10. Assume $Y, W \in \mathcal{D}_{X}, \tilde{Y}, \tilde{W} \in \mathcal{D}_{\tilde{X}}$. Define maps $Q, \tilde{Q}: \mathbb{T}^{2} \rightarrow \mathbb{R}$ by the following identities

$$
Q(\eta, \xi):=\int_{\xi}^{\eta} Y d W-Y(\xi)(W(\eta)-W(\xi))-Y^{\prime}(\xi) W^{\prime}(\xi) \mathbb{X}^{2}(\eta, \xi), \quad \eta, \xi \in \mathbb{T}
$$

and a similar expression for $\tilde{Q}$ with $Y, Y^{\prime}, W, W^{\prime}, \mathbb{X}^{2}$ replaced by $\tilde{Y}, \tilde{Y}^{\prime}, \tilde{W}, \tilde{W}^{\prime}, \tilde{\mathbb{X}}^{2}$. Then $Q, \tilde{Q} \in C_{2}^{3 \nu}$ and there exists a constant $C=C(\nu)>0$ such that for all $Y, W \in \mathcal{D}_{X}$

$$
\|Q\|_{C_{2}^{3 \nu}} \leq C\left(1+\|X\|_{C^{\nu}}+\left\|\mathbb{X}^{2}\right\|_{C_{2}^{2 \nu}}\right)\|Y\|_{\mathcal{D}_{X}}\|W\|_{\mathcal{D}_{X}}
$$

Furthermore,

$$
\begin{aligned}
\|Q-\tilde{Q}\|_{C_{2}^{3 \nu}} \leq C(1+ & \left.\|X\|_{C^{\nu}}+\left\|\mathbb{X}^{2}\right\|_{C_{2}^{2 \nu}}\right) \\
& \left(\left(\|Y\|_{\mathcal{D}_{X}}+\|\tilde{Y}\|_{\mathcal{D}_{\tilde{X}}}\right) \varepsilon_{W}+\left(\|W\|_{\mathcal{D}_{X}}+\|\tilde{W}\|_{\mathcal{D}_{\tilde{X}}}\right) \varepsilon_{Y}+\varepsilon_{X}\right) .
\end{aligned}
$$

where

$$
\begin{gathered}
\varepsilon_{Y}=\left\|Y^{\prime}-\tilde{Y}^{\prime}\right\|_{C^{\nu}}+\left\|R^{Y}-R^{\tilde{Y}}\right\|_{C_{2}^{2 \nu}}+\|Y-\tilde{Y}\|_{C^{\nu}}, \\
\varepsilon_{W}=\left\|W^{\prime}-\tilde{W}^{\prime}\right\|_{C^{\nu}}+\left\|R^{W}-R^{\tilde{W}}\right\|_{C_{2}^{2 \nu}}+\|W-\tilde{W}\|_{C^{\nu}}, \\
\varepsilon_{X}=\left(\|Y\|_{\mathcal{D}_{X}}+\|\tilde{Y}\|_{\mathcal{D}_{\tilde{X}}}\right)\left(\|W\|_{\mathcal{D}_{X}}+\|\tilde{W}\|_{\mathcal{D}_{\tilde{X}}}\right)\left(\|X-\tilde{X}\|_{C^{\nu}}+\left\|\mathbb{X}^{2}-\tilde{\mathbb{X}}^{2}\right\|_{C_{2}^{2 \nu}}\right) .
\end{gathered}
$$

Proof. See [12], Theorem 1. For formula (2.19) see [12], p.104, formula (27). 
By Lemmata 2.8 and 2.3 for any $A \in C^{2}\left(\mathbb{R}^{3}, L\left(\mathbb{R}^{3}, \mathbb{R}^{3}\right)\right), Y \in \mathcal{D}_{X}$ we can a define a map $V^{Y}: \mathbb{R}^{3} \rightarrow \mathbb{R}$ by the means of the rough path integral as follows

$$
V^{Y}(x):=\int_{0}^{1} A(x-Y(\xi)) d Y(\xi), \quad x \in \mathbb{R}^{3} .
$$

For maps $F: \mathbb{R}^{3} \rightarrow W$ and $n \geq 0$ we denote by $\|F\|_{C^{n}}$ the usual norms

$$
\|F\|_{C^{n}}=\sup _{0 \leq|\alpha| \leq n} \sup _{x \in \mathbb{R}^{3}}\left|\nabla^{\alpha} F(x)\right| .
$$

We have following bounds on the regularity of $V^{Y}$.

Lemma 2.11. There exists $C_{1}=C_{1}(\nu), C_{2}=C_{2}(\mathbb{X})$ such that for any integer $n \geq 0$ and all $Y \in \mathcal{D}_{X}, \tilde{Y} \in \mathcal{D}_{\tilde{X}}$,

$$
\left\|\nabla^{n} V^{Y}\right\|_{L^{\infty}} \leq 4 C_{1} C_{2}^{3}\left\|\nabla^{n+1} A\right\|_{C^{1}}\|Y\|_{\mathcal{D}_{X}}^{2}\left(1+\|Y\|_{\mathcal{D}_{X}}\right)
$$

and

$$
\left\|\nabla^{n} V^{Y}-\nabla^{n} V^{\tilde{Y}}\right\|_{L^{\infty}} \leq C(\nu)\|A\|_{C^{n+3}} C_{X}^{4}\left(1+\|Y\|_{\mathcal{D}_{X}}+\|\tilde{Y}\|_{\mathcal{D}_{\tilde{X}}}\right)^{3} D(Y, \tilde{Y})
$$

where

$$
C_{X}=1+\|X\|_{C^{\nu}}+\|\tilde{X}\|_{C^{\nu}}+\left\|\mathbb{X}^{2}\right\|_{C_{2}^{2 \nu}}+\left\|\tilde{\mathbb{X}}^{2}\right\|_{C_{2}^{2 \nu}}
$$

In the case of $X=\tilde{X}$, inequality (2.22) can be rewritten as

$$
\left\|\nabla^{n} V^{Y}-\nabla^{n} V^{\tilde{Y}}\right\|_{L^{\infty}} \leq 16 C_{1} C_{2}^{3}\left\|\nabla^{n+1} A\right\|_{C^{2}}\|Y\|_{\mathcal{D}_{X}}\left(1+\|Y\|_{\mathcal{D}_{X}}\right)^{2}\|Y-\tilde{Y}\|_{\mathcal{D}_{X}}^{*} .
$$

Proof. By the results [6, Lemma 7] it is known that $V^{Y} \in C^{n}$ if $A \in C^{n+2}$ and alsto that eqns. (2.21) and (2.23) hold. Now we will show (2.22). It is enough to consider the case of $n=0$. By eq. (2.18) we have

$$
\begin{aligned}
V^{Y}(x)-V^{\tilde{Y}}(x)=\quad & A(x-Y(0))(Y(1)-Y(0))-A(x-\tilde{Y}(0))(\tilde{Y}(1)-\tilde{Y}(0)) \\
& +(A(x-Y))^{\prime}(0) Y^{\prime}(0) \mathbb{X}^{2}(0,1)-(A(x-\tilde{Y}))^{\prime}(0) \tilde{Y}^{\prime}(0) \tilde{\mathbb{X}}^{2}(0,1) \\
& +Q^{x}(0,1)-\tilde{Q}^{x}(0,1),
\end{aligned}
$$

where $Q^{x}$ and $\tilde{Q}^{x}$ (given by the eq. (2.18)) satisfy inequality (2.19). Recall that $Y(1)=Y(0)$ and $\tilde{Y}(1)=\tilde{Y}(0)$, hence we have

$$
\begin{aligned}
\left|V^{Y}-V^{\tilde{Y}}\right|_{L^{\infty}} \leq & \sup _{x}\left|(A(x-Y))^{\prime}(0) Y^{\prime}(0) \mathbb{X}^{2}(0,1)-(A(x-\tilde{Y}))^{\prime}(0) \tilde{Y}^{\prime}(0) \tilde{\mathbb{X}}^{2}(0,1)\right| \\
& +\sup _{x}\left|Q^{x}(0,1)-\tilde{Q}^{x}(0,1)\right| .
\end{aligned}
$$


For the first term on the r.h.s. we have

$$
\begin{aligned}
& \left|(A(x-Y))^{\prime}(0) Y^{\prime}(0) \mathbb{X}^{2}(0,1)-(A(x-\tilde{Y}))^{\prime}(0) \tilde{Y}^{\prime}(0) \tilde{\mathbb{X}}^{2}(0,1)\right| \\
& \quad \leq\left|\left(\nabla A(x-Y(0)) Y^{\prime}(0) Y^{\prime}(0)-\nabla A(x-\tilde{Y}(0)) \tilde{Y}^{\prime}(0) \tilde{Y}^{\prime}(0)\right) \mathbb{X}^{2}(0,1)\right| \\
& \quad+\left|\nabla A(x-\tilde{Y}(0)) \tilde{Y}^{\prime}(0) \tilde{Y}^{\prime}(0)\left(\mathbb{X}^{2}(0,1)-\tilde{\mathbb{X}}^{2}(0,1)\right)\right| \\
& \quad \leq\left\|\mathbb{X}^{2}\right\|_{C_{2}^{2 \nu}}\left|\nabla A(x-Y(0)) Y^{\prime}(0) Y^{\prime}(0)-\nabla A(x-\tilde{Y}(0)) \tilde{Y}^{\prime}(0) \tilde{Y}^{\prime}(0)\right| \\
& \quad+\|A\|_{C^{2}}\left\|Y^{\prime}\right\|_{L^{\infty}}^{2}\left\|\mathbb{X}^{2}-\tilde{\mathbb{X}}^{2}\right\|_{C_{2}^{2 \nu}} \\
& \quad \leq\left\|\mathbb{X}^{2}\right\|_{C_{2}^{2 \nu}}\left\|Y^{\prime}\right\|_{L^{\infty}}^{2}\|A\|_{C^{2}}\left\|Y^{\prime}-\tilde{Y}^{\prime}\right\|_{L^{\infty}}+\left\|\mathbb{X}^{2}\right\|_{C_{2}^{2 \nu}}\|A\|_{C^{1}}\left(\left\|Y^{\prime}\right\|_{L^{\infty}}+\left\|\tilde{Y}^{\prime}\right\|_{L^{\infty}}\right)\left\|Y^{\prime}-\tilde{Y}^{\prime}\right\|_{L^{\infty}} \\
& \quad+\|A\|_{C^{2}}\left\|Y^{\prime}\right\|_{L^{\infty}}^{2}\left\|\mathbb{X}^{2}-\tilde{\mathbb{X}}^{2}\right\|_{C_{2}^{2 \nu}} .
\end{aligned}
$$

By (2.19) we can estimate second term as follows

$$
\begin{aligned}
\left|Q^{x}-\tilde{Q}^{x}\right|_{C_{2}^{3 \nu}} & \leq C\left[\left(\|A(x-Y)\|_{\mathcal{D}_{X}}+\|A(x-\tilde{Y})\|_{\mathcal{D}_{\tilde{X}}}\right) \varepsilon_{Y}\right. \\
& \left.+\left(\|Y\|_{\mathcal{D}_{X}}+\|\tilde{Y}\|_{\mathcal{D}_{\tilde{X}}}\right) \varepsilon_{A}+\varepsilon_{X}\right]
\end{aligned}
$$

where

$$
\begin{aligned}
\varepsilon_{Y} & =\|Y-\tilde{Y}\|_{C^{\nu}}+\left\|Y^{\prime}-\tilde{Y}^{\prime}\right\|_{C^{\nu}}+\left\|R^{Y}-R^{\tilde{Y}}\right\|_{C_{2}^{2 \nu}}, \\
\varepsilon_{A} & =\|A(x-Y)-A(x-\tilde{Y})\|_{C^{\nu}}+\left\|A(x-Y)^{\prime}-A(x-\tilde{Y})^{\prime}\right\|_{C^{\nu}} \\
& +\left\|R^{A(x-Y)}-R^{A(x-\tilde{Y})}\right\|_{C_{2}^{2 \nu}}, \\
\varepsilon_{X} & =\left(\|A(x-Y)\|_{\mathcal{D}_{X}}+\|A(x-\tilde{Y})\|_{\mathcal{D}_{\tilde{X}}}\right) \\
& \times\left(\|Y\|_{\mathcal{D}_{X}}+\|\tilde{Y}\|_{\mathcal{D}_{\tilde{X}}}\right)\left(|X-\tilde{X}|_{C^{\nu}}+\left|\mathbb{X}^{2}-\tilde{\mathbb{X}}^{2}\right|_{C_{2}^{2 \nu}}\right) .
\end{aligned}
$$

By formula (2.8) we can estimate $\varepsilon_{A}$ as follows

$$
\begin{aligned}
\varepsilon_{A} & \leq K\|A\|_{C^{3}}\left(1+\|X\|_{C^{\nu}}+\|\tilde{X}\|_{C^{\nu}}\right)^{3}\left(1+\|Y\|_{D_{X}}+\|\tilde{Y}\|_{D_{\tilde{X}}}\right)^{2} \\
& \times\left(\|X-\tilde{X}\|_{C^{\nu}}+\|Y-\tilde{Y}\|_{C^{\nu}}+\left\|Y^{\prime}-\tilde{Y}^{\prime}\right\|_{C^{\nu}}+\left\|R^{Y}-R^{\tilde{Y}}\right\|_{C_{2}^{2 \nu}}\right) .
\end{aligned}
$$

By inequality (2.7) we infer that

$$
\|A(x-Y)\|_{\mathcal{D}_{X}} \leq K\|A\|_{C^{2}}\|Y\|_{D_{X}}\left(1+\|Y\|_{D_{X}}\right)\left(1+\|X\|_{C^{\nu}}\right)^{2},
$$

and similarly,

$$
\|A(x-\tilde{Y})\|_{\mathcal{D}_{\tilde{X}}} \leq K\|A\|_{C^{2}}\|\tilde{Y}\|_{D_{\tilde{X}}}\left(1+\|\tilde{Y}\|_{D_{\tilde{X}}}\right)\left(1+\|\tilde{X}\|_{C^{\nu}}\right)^{2} .
$$

Therefore, combining inequalities (2.26) with (2.26), (2.27) and (2.28) we get

$$
\begin{gathered}
\left\|Q^{x}-\tilde{Q}^{x}\right\|_{C_{2}^{3 \nu}} \leq C(\nu)\|A\|_{C^{n+3}}\left(1+\|X\|_{C^{\nu}}+\|\tilde{X}\|_{C^{\nu}}\right)^{4}\left(1+\|Y\|_{\mathcal{D}_{X}}+\|\tilde{Y}\|_{\mathcal{D}_{\tilde{X}}}\right)^{3} \\
\left(\|X-\tilde{X}\|_{C^{\nu}}+\left\|\mathbb{X}^{2}-\tilde{\mathbb{X}}^{2}\right\|_{C_{2}^{2 \nu}}+\left\|Y^{\prime}-\tilde{Y}^{\prime}\right\|_{C^{\nu}}+\left\|R^{Y}-R^{\tilde{Y}}\right\|_{C_{2}^{2 \nu}}+\|Y-\tilde{Y}\|_{C^{\nu}}\right) .
\end{gathered}
$$

Hence, the result follows from (2.25) and (2.29). 


\section{The Evolution Problem for a Rough Filament}

Let $\mathcal{D}_{\mathbb{X}, T}=C\left([0, T], \mathcal{D}_{X}\right)$, where the space $\mathcal{D}_{X}$ has been defined in Definition 2.2, be a vector space with the usual supremum norm

$$
\|F\|_{\mathcal{D}_{\mathbb{X}, T}}=\sup _{t \in[0, T]}\|F(t)\|_{\mathcal{D}_{X}}^{*}
$$

Obviously $\mathcal{D}_{\mathbb{X}, T}$ is a Banach space. For an element $\gamma \in \mathcal{D}_{\mathbb{X}, T}$, the time-dependent vector field

$$
(t, x) \mapsto u^{\gamma(t)}(x)=\int_{0}^{1} \nabla \phi(x-\gamma(t, \xi)) \times d \gamma(t, \xi)
$$

is well defined by the rough integral and it is meaningful to consider the Cauchy problem (1.1) in $\mathcal{D}_{\mathbb{X}, T}$. The following local existence and uniqueness result been proved in [6], see Theorem 3, p. 1842 .

Theorem 3.1. Assume $\phi \in C^{6}\left(\mathbb{R}^{3}, \mathbb{R}\right), \nu \in(1 / 3,1), \mathbb{X}=\left(X, \mathbb{X}^{2}\right)$ is a $\nu$-rough path, $\gamma_{0} \in \mathcal{D}_{X}$. Then there exists a time $T_{0}=T_{0}\left(\nu,\|\phi\|_{C^{5}}, \mathbb{X}\right)>0$ such that the problem (1.1) has unique solution in the space $\mathcal{D}_{\gamma_{0}, T_{0}} \subset \mathcal{D}_{\mathbb{X}, T_{0}}$. Moveover if $\mathbb{X}, \tilde{\mathbb{X}}$ are two $\nu$-rough paths, $\gamma_{0} \in \mathcal{D}_{X}$, $\tilde{\gamma}_{0} \in \mathcal{D}_{\tilde{X}}$ and $\gamma, \tilde{\gamma}$ two solutions of (1.1) respectively in $\mathcal{D}_{\mathbb{X}, T_{0}}$ and $\mathcal{D}_{\tilde{\mathbb{X}}, \tilde{T}_{0}}$ then there exists a constant $C=C\left(\mathbb{X}, \tilde{\mathbb{X}}, \phi, \nu,\left\|\gamma_{0}\right\|_{\mathcal{D}_{X}}^{*},\left\|\tilde{\gamma}_{0}\right\|_{\mathcal{D}_{\tilde{X}}}^{*}\right)$ such that

$$
\sup _{0 \leq t \leq \min \left(T_{0}, \tilde{T}_{0}\right)} D(\gamma(t), \tilde{\gamma}(t)) \leq C D(\gamma(0), \tilde{\gamma}(0)) .
$$

This result can be easily proven by applying the Banach Fixed Point Theorem in the space $\mathcal{D}_{\mathbb{X}, T}$ with a sufficiently small $T$. The requirements on $\phi$ can be easily understood as follows. In order for $u^{\gamma}$ to be well defined the function $\nabla \phi$ has to be twice differentiable. Moreover in order to apply the fixed point theorem the function $(t, \xi) \mapsto u^{\gamma(t)}(\gamma(t, \xi))$ has to belong to $\mathcal{D}_{\mathbb{X}, T}$ and this can enforced by requiring that the map $x \mapsto u^{\gamma(t)}(x)$ has to be $C^{2}$ in space, uniformly in time. This requires $\nabla \phi$ to be at least 4 times differentiable. Furthermore we need a strict contraction of the fixed-point map which holds at the price of an additional derivative of $\nabla \phi$ ending up with the requirement that $\phi$ has to be six times differentiable. It is not obvious how to improve on this requirement for a general rough initial data and $\nu \in(1 / 3,1 / 2)$.

The arguments used in Theorem 3.1 does not allow to have a global solution. The aim of the present paper is to enforce further structure condition on the kernel $\phi$ in order to be able to use the energy method introduced in [5].

To prove the global existence result for the problem (1.1) we need the following additional hypothesis

Hypothesis 3.2. Assume that the function $\phi$ appearing in eq. (1.2) has a Fourier transform $\hat{\phi}: \mathbb{R}^{3} \rightarrow \mathbb{R}$ which is real, even and non-negative and satisfying the integrability condition

$$
\int_{\mathbb{R}^{3}}\left(1+|k|^{2}\right)^{2} \hat{\phi}(k) d k<\infty .
$$

Example 3.3. The function $\phi_{\mu}, \mu>0$ defined by

$$
\phi_{\mu}(\cdot)=\frac{1}{\left(|\cdot|^{2}+\mu^{2}\right)^{\frac{1}{2}}}
$$


is smooth and satisfies Hypothesis 3.2, see p.6 of [5]. This function is also of $C^{\infty}$-class so it satisfy also the assumptions of Theorem 3.1 and as a result is an explicit example which satisfy all the assumptions needed for the following global existence result.

Theorem 3.4. Assume that $\gamma_{0}$ is a geometric $\nu$-rough path, $\phi \in C^{6}\left(\mathbb{R}^{3}, \mathbb{R}\right)$ satisfies Hypothesis 3.2 and $\nu \in(1 / 3,1)$. Then for every $T>0$, the problem (1.1) has unique solution in $\mathcal{D}_{\gamma_{0}, T}$.

We will need the following definition.

Definition 3.5. Let $\phi \in C^{4}\left(\mathbb{R}^{3}, \mathbb{R}\right), \gamma \in \mathcal{D}_{X}$. Let

$$
\psi^{\gamma}(x)=\int_{0}^{1} \phi(x-\gamma(\eta)) d \gamma(\eta)
$$

and

$$
\mathcal{H}_{X}^{\phi}(\gamma)=\frac{1}{2} \int_{0}^{1} \psi^{\gamma}(\gamma(\xi)) \cdot d \gamma(\xi)=\frac{1}{2} \int_{0}^{1} \int_{0}^{1} \phi(\gamma(\xi)-\gamma(\eta))(d \gamma(\xi) \cdot d \gamma(\eta)) .
$$

The quantity $\mathcal{H}_{X}^{\phi}(\gamma)$ is called the energy of path $\gamma$. We will omit $\phi$ below.

Remark 3.6. Definition (3.2) is well posed. Indeed, by Lemma $2.11 \psi^{\gamma} \in C^{2}\left(\mathbb{R}^{3}, \mathbb{R}^{3}\right)$ and, therefore, it follows by Lemma 2.3 that $\psi^{\gamma} \circ \gamma \in \mathcal{D}_{X}$. Moreover, if $\nu>1 / 2$ and $\gamma \in C^{1}\left(\mathbb{T}, \mathbb{R}^{3}\right)$, then by Remark 2.9 the line integrals in the definition of the energy are understood in the sense of Young.

Lemma 3.7. Assume that $\phi \in C^{4}\left(\mathbb{R}^{3}, \mathbb{R}\right)$. Then there exists a constant $C=C(\nu, \mathbb{X})$ such that for all $\gamma \in \mathcal{D}_{X}$

$$
\left|\mathcal{H}_{X}(\gamma)\right| \leq C\|\phi\|_{C^{4}}\|\gamma\|_{\mathcal{D}_{X}}^{4}\left(1+\|\gamma\|_{\mathcal{D}_{X}}\right)^{2} .
$$

Furthermore, for any $R>0$ there exists $C=C(R)$ such that for any $\gamma \in \mathcal{D}_{X}, \tilde{\gamma} \in \mathcal{D}_{\tilde{X}}$ satisfying

we have

$$
\begin{gathered}
\|\gamma\|_{\mathcal{D}_{X}} \leq R,\|\tilde{\gamma}\|_{\mathcal{D}_{\tilde{X}}} \leq R \\
C_{X, \tilde{X}}=\|X\|_{C^{\nu}}+\|\tilde{X}\|_{C^{\nu}}+\left\|\mathbb{X}^{2}\right\|_{C_{2}^{2 \nu}}+\left\|\tilde{\mathbb{X}}^{2}\right\|_{C_{2}^{2 \nu}}<R
\end{gathered}
$$

$$
\left|\mathcal{H}_{X}(\gamma)-\mathcal{H}_{\tilde{X}}(\tilde{\gamma})\right| \leq C(R) D(\gamma, \tilde{\gamma})
$$

In particular the map $\mathcal{H}_{X}: \mathcal{D}_{X} \rightarrow \mathbb{R}$ is Lipshitz on balls, i.e. for any $R>0$ there exists $C=C(R)$ such that for any $\gamma, \tilde{\gamma} \in \mathcal{D}_{X}:\|\gamma\|_{\mathcal{D}_{X}} \leq R,\|\tilde{\gamma}\|_{\mathcal{D}_{X}} \leq R$ we have

$$
\left|\mathcal{H}_{X}(\gamma)-\mathcal{H}_{X}(\tilde{\gamma})\right| \leq C(R)\|\gamma-\tilde{\gamma}\|_{\mathcal{D}_{X}}^{*}
$$

Proof. First we will show inequality (3.3). By representation (2.17) we have

$$
\begin{aligned}
\mathcal{H}_{X}(\gamma) & =\frac{1}{2}\left(\psi^{\gamma}(\gamma(0))(\gamma(1)-\gamma(0))\right)+\left[\nabla \psi^{\gamma}(\gamma(0)) \gamma^{\prime}(0)\right] \gamma^{\prime}(0) \mathbb{X}^{2}(1,0)+Q(0,1) \\
& =I+I I+I I I .
\end{aligned}
$$

Since $\gamma(1)=\gamma(0)$ we infer that $I=0$. Concerning the second term by Lemma 2.11 we have the following estimate

$$
|I I| \leq\left\|\mathbb{X}^{2}\right\|_{C_{2}^{2 \nu}}\left\|\nabla \psi^{\gamma}\right\|_{L^{\infty}}\left\|\gamma^{\prime}\right\|_{L^{\infty}}^{2} \leq C(\nu, \mathbb{X})\|\phi\|_{C^{3}}\|\gamma\|_{\mathcal{D}_{X}}^{4}\left(1+\|\gamma\|_{\mathcal{D}_{X}}\right) .
$$

For third term, from inequality (2.18) we infer that

$$
|I I I| \leq\|Q\|_{C_{2}^{3 \nu}} \leq C(\nu, \mathbb{X})\left\|\psi^{\gamma}(\gamma)\right\|_{\mathcal{D}_{X}}\|\gamma\|_{\mathcal{D}_{X}} .
$$


Then by Lemmata 2.3 and 2.11 we have

$$
\begin{aligned}
\left\|\psi^{\gamma}(\gamma)\right\|_{\mathcal{D}_{X}} & \leq C(\nu, \mathbb{X})\left\|\psi^{\gamma}\right\|_{C^{2}}\|\gamma\|_{\mathcal{D}_{X}}\left(1+\|\gamma\|_{\mathcal{D}_{X}}\right) \\
& \leq C(\nu, \mathbb{X})\|\phi\|_{C^{4}}\|\gamma\|_{\mathcal{D}_{X}}^{3}\left(1+\|\gamma\|_{\mathcal{D}_{X}}\right)^{2} .
\end{aligned}
$$

Combining inequalities (3.7), (3.8) and (3.9) we get inequality (3.3). To prove inequality (3.4) we start by formula (2.18) to get

$$
\begin{aligned}
\mathcal{H}_{X}(\gamma)-\mathcal{H}_{\tilde{X}}(\tilde{\gamma}) & =\frac{1}{2}\left[\left(\nabla \psi^{\gamma}(\gamma(0)) \gamma^{\prime}(0) \gamma^{\prime}(0)-\nabla \psi \tilde{\gamma}(\tilde{\gamma}(0)) \tilde{\gamma}^{\prime}(0) \tilde{\gamma}^{\prime}(0)\right) \mathbb{X}^{2}(1,0)\right. \\
& \left.+\nabla \psi^{\tilde{\gamma}}(\tilde{\gamma}(0)) \tilde{\gamma}^{\prime}(0) \tilde{\gamma}^{\prime}(0)\left(\mathbb{X}^{2}(1,0)-\tilde{\mathbb{X}}^{2}(1,0)\right)+Q(0,1)-\tilde{Q}(0,1)\right] \\
& =: I+I I+I I I
\end{aligned}
$$

The first term in (3.10) can be represented as follows

$$
\begin{aligned}
I & =\left(\nabla \psi^{\gamma}(\gamma(0)) \gamma^{\prime}(0) \gamma^{\prime}(0)-\nabla \psi^{\tilde{\gamma}}(\tilde{\gamma}(0)) \tilde{\gamma}^{\prime}(0) \tilde{\gamma}^{\prime}(0)\right) \mathbb{X}^{2}(1,0) \\
& =\left[\left(\nabla \psi^{\gamma}(\gamma(0))-\nabla \psi^{\tilde{\gamma}}(\tilde{\gamma}(0))\right) \gamma^{\prime}(0) \gamma^{\prime}(0)\right. \\
& +\nabla \psi^{\tilde{\gamma}}(\tilde{\gamma}(0))\left(\gamma^{\prime}(0)-\tilde{\gamma}(0)\right) \gamma^{\prime}(0) \\
& \left.+\nabla \psi^{\tilde{\gamma}}(\tilde{\gamma}(0)) \tilde{\gamma}(0)\left(\gamma^{\prime}(0)-\tilde{\gamma}(0)\right)\right] \mathbb{X}^{2}(1,0)=A+B+C,
\end{aligned}
$$

and the first term in (3.11) can be estimated as follows

$$
\begin{aligned}
|A| & =\left|\left(\nabla \psi^{\gamma}(\gamma(0))-\nabla \psi^{\tilde{\gamma}}(\tilde{\gamma}(0))\right) \gamma^{\prime}(0) \gamma^{\prime}(0) \mathbb{X}^{2}(1,0)\right| \\
& \leq\left\|\mathbb{X}^{2}\right\|_{C_{2}^{2 \nu}}\|\gamma\|_{\mathcal{D}_{X}}^{2}\left(\mid \nabla \psi^{\gamma}(\gamma(0))\right. \\
& \left.-\nabla \psi^{\gamma}(\tilde{\gamma}(0))|+| \nabla \psi^{\gamma}(\tilde{\gamma}(0))-\nabla \psi^{\tilde{\gamma}}(\tilde{\gamma}(0)) \mid\right) \\
& \leq\left\|\mathbb{X}^{2}\right\|_{C_{2}^{2 \nu}}\|\gamma\|_{\mathcal{D}_{X}}^{2}\left(\left\|\psi^{\gamma}\right\|_{C^{2}}|\gamma(0)-\tilde{\gamma}(0)|\right. \\
& +C_{X}^{4}\|\phi\|_{C^{4}}\left(1+\|\gamma\|_{\mathcal{D}_{X}}+\|\tilde{\gamma}\|_{\mathcal{D}_{\tilde{X}}}\right)^{3} D(\gamma, \tilde{\gamma}) \\
& \leq K C_{X}^{4}\|\phi\|_{C^{4}}\left(1+\|\gamma\|_{\mathcal{D}_{X}}+\|\tilde{\gamma}\|_{\mathcal{D}_{\tilde{X}}}\right)^{3} D(\gamma, \tilde{\gamma}) .
\end{aligned}
$$

Here the second inequality follows from inequality (2.22) and the third one from inequality (2.21). For second term in (3.11) we have by inequality (2.21)

$$
\begin{aligned}
|B| & \leq C\left\|\mathbb{X}^{2}\right\|_{C_{2}^{2 \nu}}\|\gamma\|_{\mathcal{D}_{X}}\|\phi\|_{C^{3}}\|\tilde{\gamma}\|_{\mathcal{D}_{X}}^{2}\left(1+\|\tilde{\gamma}\|_{\mathcal{D}_{X}}\right) D(\gamma, \tilde{\gamma}) \\
& \leq C C_{X}\left(1+\|\gamma\|_{\mathcal{D}_{X}}+\|\tilde{\gamma}\|_{\mathcal{D}_{\tilde{X}}}\right)^{3} D(\gamma, \tilde{\gamma}) .
\end{aligned}
$$

Similarly, we have for third term

$$
|C| \leq C\left(\nu, \mathbb{X},\|\gamma\|_{\mathcal{D}_{X}},\|\tilde{\gamma}\|_{\mathcal{D}_{X}}\right) D(\gamma, \tilde{\gamma}) .
$$

Going back to the term $I I$ in (3.10) we observe that it can be estimated as follows

$$
|I I| \leq\left\|\nabla \psi^{\tilde{\gamma}}\right\|_{L^{\infty}}\|\tilde{\gamma}\|_{\mathcal{D}_{X}}^{2} D(\gamma, \tilde{\gamma}) \leq C_{X}^{3}\|\phi\|_{C^{3}}\left(1+\|\gamma\|_{\mathcal{D}_{X}}+\|\tilde{\gamma}\|_{\mathcal{D}_{\tilde{X}}}\right)^{3} D(\gamma, \tilde{\gamma}) .
$$


Thus it remains to estimate third term of equality (3.10). By inequality (2.19) we have

$$
\begin{aligned}
|Q(0,1)-\tilde{Q}(0,1)| & \leq\|Q-\tilde{Q}\|_{C_{2}^{3 \nu}} \\
& \leq C_{X}\left[\left(\left\|\psi^{\tilde{\gamma}}(\tilde{\gamma})\right\|_{\mathcal{D}_{\tilde{X}}}+\left\|\psi^{\gamma}(\gamma)\right\|_{\mathcal{D}_{X}}\right) D(\gamma, \tilde{\gamma})\right. \\
& +\left(\|\tilde{\gamma}\|_{\mathcal{D}_{\tilde{X}}}+\|\tilde{\gamma}\|_{\mathcal{D}_{\tilde{X}}}\right) D\left(\psi^{\tilde{\gamma}}(\tilde{\gamma}), \psi^{\gamma}(\gamma)\right) \\
& +\left(\left\|\psi^{\tilde{\gamma}}(\tilde{\gamma})\right\|_{\mathcal{D}_{\tilde{X}}}+\left\|\psi^{\gamma}(\gamma)\right\|_{\mathcal{D}_{X}}\right)\left(\|\tilde{\gamma}\|_{\mathcal{D}_{\tilde{X}}}+\|\tilde{\gamma}\|_{\mathcal{D}_{\tilde{X}}}\right) \\
& \times\left(\|X-\tilde{X}\|_{C^{\nu}}+\left\|\mathbb{X}^{2}-\tilde{\mathbb{X}}^{2}\right\|_{\left.\left.C_{2}^{2 \nu}\right)\right]}\right.
\end{aligned}
$$

By inequality (2.21), the term $\left\|\psi^{\tilde{\gamma}}(\tilde{\gamma})\right\|_{\mathcal{D}_{X}}$ is bounded by the constant $C=C\left(\nu, \mathbb{X},\|\tilde{\gamma}\|_{\mathcal{D}_{X}}\right)$. Therefore, to prove estimate (3.5) it is enough to show that there exists a constant $C=$ $C(\nu, \mathbb{X}, R)$ such that for $\gamma, \tilde{\gamma} \in \mathcal{D}_{X}$ with $\|\gamma\|_{\mathcal{D}_{X}},\|\tilde{\gamma}\|_{\mathcal{D}_{X}} \leq R$

$$
D\left(\psi^{\tilde{\gamma}}(\tilde{\gamma}), \psi^{\gamma}(\gamma)\right) \leq C D(\gamma, \tilde{\gamma})
$$

By the triangle inequality we have

$$
\begin{aligned}
D\left(\psi^{\tilde{\gamma}}(\tilde{\gamma}), \psi^{\gamma}(\gamma)\right) & \leq D\left(\psi^{\tilde{\gamma}}(\tilde{\gamma}), \psi^{\tilde{\gamma}}(\gamma)\right)+D\left(\psi^{\tilde{\gamma}}(\gamma), \psi^{\gamma}(\gamma)\right) \\
& =I+I I
\end{aligned}
$$

The first term can be estimated by using inequality (2.8) as follows

$$
|I| \leq K C_{X}^{3}\left\|\psi^{\tilde{\gamma}}\right\|_{C^{3}}\left(1+\|\tilde{\gamma}\|_{\mathcal{D}_{\tilde{X}}}+\|\gamma\|_{\mathcal{D}_{X}}\right)^{2} D(\gamma, \tilde{\gamma}) .
$$

By inequality (2.21) we have

$$
\left\|\psi^{\tilde{\gamma}}\right\|_{C^{3}} \leq C\|\phi\|_{C^{5}}\|\tilde{\gamma}\|_{\mathcal{D}_{X}}^{2}\left(1+\|\tilde{\gamma}\|_{\mathcal{D}_{X}}\right)
$$

Combining inequalities (3.17) and (3.18) we obtain the necessary estimate for $I$. It remains to find an estimate for the term $I I$. By inequalities (2.7) and (2.23) we have

$$
\begin{aligned}
I I & =D\left(\psi^{\tilde{\gamma}}(\gamma), \psi^{\gamma}(\gamma)\right) \leq\left(1+\|X\|_{\nu}\right)\left\|\psi^{\tilde{\gamma}}(\gamma)-\psi^{\gamma}(\gamma)\right\|_{\mathcal{D}_{X}} \\
& \leq K\left\|\nabla \psi^{\tilde{\gamma}}-\nabla \psi^{\gamma}\right\|_{C^{1}}\|\gamma\|_{\mathcal{D}_{X}}\left(1+\|\gamma\|_{\mathcal{D}_{X}}\right)\left(1+\|X\|_{\nu}\right)^{3} \\
& \leq K\|\phi\|_{C^{5}} C_{X}^{7}\left(1+\|\gamma\|_{\mathcal{D}_{X}}+\|\tilde{\gamma}\|_{\mathcal{D}_{\tilde{X}}}\right)^{5} D(\gamma, \tilde{\gamma}) .
\end{aligned}
$$

Hence the inequality (3.4) follows. Finally the bound (3.5) is a consequence of eq. (3.4).

Let us recall the definition (1.2) of the vector field $u^{\gamma}$ generated by a controlled path $\gamma$ :

$$
u^{\gamma}(x)=\int_{0}^{1} \nabla \phi(x-\gamma(\xi)) \times d \gamma(\xi), \quad \gamma \in \mathcal{D}_{X} .
$$

Now we will show that if the energy functional of $\gamma$ is bounded then the associated velocity field is a smooth function. We have

Lemma 3.8. Assume that $\phi \in C^{4}\left(\mathbb{R}^{3}, \mathbb{R}\right)$ and Hypothesis 2.6 holds. Then the energy function $\mathcal{H}_{X}: \mathcal{D}_{X} \rightarrow \mathbb{R}$ is continuous. Furthermore if $\gamma$ is a geometric rough path then

$$
\mathcal{H}_{X}(\gamma)=\frac{1}{(2 \pi)^{3}} \int_{\mathbb{R}^{3}} \hat{\phi}(k)\left|\int_{0}^{1} e^{i(k, \gamma(\xi))} d \gamma(\xi)\right|^{2} d k \geq 0 .
$$


Moreover, if in addition the integral $\int_{\mathbb{R}^{3}}|k|^{2(1+n)} \hat{\phi}(k) d k$ is finite and $\phi \in C^{n+4}\left(\mathbb{R}^{3}, \mathbb{R}^{3}\right)$, then for any $n \in \mathbb{N} 0$, we have following bound

$$
\left\|\nabla^{n} u^{\gamma}\right\|_{L^{\infty}}^{2} \leq \frac{1}{(2 \pi)^{3}}\left[\int_{\mathbb{R}^{3}}|k|^{2(1+n)} \hat{\phi}(k) d k\right] \mathcal{H}_{X}(\gamma), \quad \gamma \in \mathcal{D}_{X} .
$$

provided that

Proof. For a smooth curve $\gamma$ Lemma 3.8 has been proved in [5, Lemma 3]. In the general case, when $\gamma \in \mathcal{D}_{X}$, it is enough to notice that both sides of eq. (3.21) and of inequality (3.22) are locally Lipshitz and therefore, continuous w.r.t. distance $D(Y, \tilde{Y}), Y \in \mathcal{D}_{X}, \tilde{Y} \in \mathcal{D}_{\tilde{X}}$ defiend in (2.16). Indeed the continuity of $\mathcal{H}_{X}$ and continuity of $\left\|\nabla^{n} u^{\gamma}\right\|_{L^{\infty}}$ readily follows from Lemma 2.11 .

Now we are going to show that energy is a local integral of motion for problem (1.1).

Lemma 3.9. Let $\gamma_{0}$ a geometric $\nu$-rough path and $\gamma \in \mathcal{D}_{\gamma_{0}, T_{0}}$ be a local solution of problem (1.1) (such a solution exists by Theorem 3.1). Then

$$
\frac{d \mathcal{H}_{\gamma_{0}}(\gamma(s))}{d s}=0, \quad s \in\left[0, T_{0}\right) .
$$

Proof. Since $\gamma(0)=\gamma_{0} \in \mathcal{D}_{\gamma_{0}}$ is a geometric rough path (we will denote its area component by $\left.\Gamma_{0}\right)$ there exist sequence $\left\{\gamma_{0}^{n}\right\}_{n=1}^{\infty}$ of piecewise smooth closed curves in $\mathbb{R}^{3}$ such that if we denote by $\left(\gamma_{0}^{n}, \Gamma_{0}^{n}\right)$ their canonical lift to the space of $\nu$-rough paths we have

$$
\left\|\gamma_{0}^{n}-\gamma_{0}\right\|_{C^{\nu}}+\left\|\Gamma_{0}^{n}-\Gamma_{0}\right\|_{C_{2}^{2 \nu}} \rightarrow 0, \quad n \rightarrow \infty .
$$

Now observe that $\gamma_{0}^{n} \in \mathcal{D}_{\gamma_{0}^{n}}, \gamma_{0} \in \mathcal{D}_{\gamma_{0}}$ since we can take $\left(\gamma_{0}^{n}\right)^{\prime}=\left(\gamma_{0}\right)^{\prime}=1$ and $R^{\gamma_{0}^{n}}=R^{\gamma_{0}}=$ 0 . Hence we deduce that

$$
D\left(\gamma_{0}^{n}, \gamma_{0}\right) \rightarrow 0, \quad n \rightarrow \infty
$$

Denote by $\gamma^{n} \in C\left([0, \infty), \mathbf{H}^{1}\left(\mathbb{T}, \mathbb{R}^{3}\right)\right)$ the global solution of problem (1.1) with initial condition $\gamma_{0}^{n}$. Existence of such solution has been proved in Theorem 2 of [5]. Moreover note that for smooth functions $\gamma^{n}$ controlled by the rough path $\left(\gamma_{0}^{n}, \Gamma_{0}^{n}\right)$ the integral defined via rough paths coincide with the standard Lebesgue integral and the solution of the Cauchy problem in $C\left([0, \infty), \mathbf{H}^{1}\left(\mathbb{T}, \mathbb{R}^{3}\right)\right)$ with the rough solution whose local existence is stated in Theorem 3.1. The locally Lipshitz dependence of the rough solution on the initial data stated in Theorem 3.1 implies that

$$
\lim _{n \rightarrow \infty} \sup _{t \in\left[0, T_{0}\right]} D\left(\gamma^{n}(t), \gamma(t)\right) \lesssim \lim _{n \rightarrow \infty} D\left(\gamma^{n}(0), \gamma(0)\right)=0
$$

for a stricly positive $T_{0}$ which depends only on the rough path norm of $\gamma_{0}$. Therefore, by the continuity of the energy functional $\mathcal{H}_{\gamma_{0}}$ we have

$$
\mathcal{H}_{\gamma_{0}}(\gamma(s))=\lim _{n \rightarrow \infty} \mathcal{H}_{\gamma_{0}^{n}}\left(\gamma^{n}(s)\right), \quad s \in\left[0, T_{0}\right] .
$$

Furthermore, by Lemma 2 of [5], we have

$$
\mathcal{H}_{\gamma_{0}^{n}}\left(\gamma^{n}(s)\right)=\mathcal{H}_{\gamma_{0}^{n}}\left(\gamma_{0}^{n}\right), \quad s \in\left[0, T_{0}\right]
$$

As a result, combining inequalities (3.23) and (3.24) we get the statement of the Lemma.

Now we are ready to prove Theorem 3.4. 
Proof of Theorem 3.4. According to Theorem 3.1 there exists a unique local solution of problem (1.1). Then, we can find $T^{*}>0$ and a unique maximal local solution $\gamma:\left[0, T^{*}\right) \rightarrow \mathcal{D}_{\gamma_{0}}$ which then satisfies

$$
\lim _{t \nearrow T^{*}}\|\gamma(t)\|_{\mathcal{D}_{\gamma_{0}}}=\infty
$$

We need to show that $T^{*}=\infty$. Therefore, it is enough to prove

$$
\sup _{t \in\left[0, T^{*}\right)}\|\gamma(t)\|_{\mathcal{D}_{\gamma_{0}}}<\infty .
$$

Indeed, by contradiction with (3.25), the result will follow. In the rest of the proof we show such estimate. Notice that we will have

$$
\mathcal{H}_{\gamma_{0}}(\gamma(s))=\mathcal{H}_{\gamma_{0}}\left(\gamma_{0}\right), \quad s \in\left[0, T^{*}\right)
$$

and recall that

$$
\gamma(t)=\gamma_{0}+\int_{0}^{t} u^{\gamma(s)}(\gamma(s)) d s
$$

Firstly we have

$$
\begin{aligned}
\|\gamma(t)\|_{L^{\infty}} & \leq\left\|\gamma_{0}\right\|_{L^{\infty}}+\int_{0}^{t}\left\|u^{\gamma(s)}\right\|_{L^{\infty}} d s \leq\left\|\gamma_{0}\right\|_{L^{\infty}}+C \int_{0}^{t} \mathcal{H}_{\gamma_{0}}^{1 / 2}(\gamma(s)) d s \\
& \leq\left\|\gamma_{0}\right\|_{L^{\infty}}+C \mathcal{H}_{\gamma_{0}}^{1 / 2}\left(\gamma_{0}\right) t, \quad t \in\left[0, T^{*}\right) .
\end{aligned}
$$

It follows from (3.27) that

$$
\gamma^{\prime}(t)=\gamma_{0}^{\prime}+\int_{0}^{t} \nabla u^{\gamma(s)}(\gamma(s)) \gamma^{\prime}(s) d s, t \in\left[0, T^{*}\right) .
$$

Therefore, by Lemmata 3.9 and 3.8 we have

$$
\begin{aligned}
\left\|\gamma^{\prime}(t)\right\|_{L^{\infty}} & \leq\left\|\gamma_{0}^{\prime}\right\|_{L^{\infty}}+\int_{0}^{t}\left\|\nabla u^{\gamma(s)}\right\|_{L^{\infty}}\left\|\gamma^{\prime}(s)\right\|_{L^{\infty}} d s \leq\left\|\gamma_{0}^{\prime}\right\|_{L^{\infty}}+\int_{0}^{t} C \mathcal{H}_{\gamma_{0}}^{1 / 2}(\gamma(s))\left\|\gamma^{\prime}(s)\right\|_{L^{\infty}} d s \\
& =\left\|\gamma_{0}^{\prime}\right\|_{L^{\infty}}+\int_{0}^{t} C \mathcal{H}_{\gamma_{0}}^{1 / 2}\left(\gamma_{0}\right)\left\|\gamma^{\prime}(s)\right\|_{L^{\infty}} d s, \quad t \in\left[0, T^{*}\right) .
\end{aligned}
$$

Then by the Gronwall Lemma we infer our second estimate

$$
\left\|\gamma^{\prime}(t)\right\|_{L^{\infty}} \leq\left\|\gamma_{0}^{\prime}\right\|_{L^{\infty}} e^{C \mathcal{H}_{\gamma_{0}}^{1 / 2}\left(\gamma_{0}\right) t}, \quad t \in\left[0, T^{*}\right) .
$$

We will need one more auxiliary estimate. We have

$$
\begin{aligned}
\|\gamma(t)\|_{C^{\nu}} & \leq\left\|\gamma_{0}\right\|_{C^{\nu}}+\int_{0}^{t}\left\|u^{\gamma(s)}(\gamma(s))\right\|_{C^{\nu}}\|d s \leq\| \gamma_{0}\left\|_{C^{\nu}}+\int_{0}^{t}\right\| \nabla u^{\gamma(s)}\left\|_{L^{\infty}}\right\| \gamma(s) \|_{C^{\nu}} d s \\
& \leq\left\|\gamma_{0}\right\|_{C^{\nu}}+\int_{0}^{t} C \mathcal{H}_{\gamma_{0}}^{1 / 2}(\gamma(s))\|\gamma(s)\|_{C^{\nu}} d s \\
& =\left\|\gamma_{0}\right\|_{C^{\nu}}+\int_{0}^{t} C \mathcal{H}_{\gamma_{0}}^{1 / 2}\left(\gamma_{0}\right)\|\gamma(s)\|_{C^{\nu}} d s, \quad t \in\left[0, T^{*}\right) .
\end{aligned}
$$

Thus, by the Gronwall Lemma we get

$$
\|\gamma(t)\|_{C^{\nu}} \leq\left\|\gamma_{0}\right\|_{C^{\nu}} e^{C \mathcal{H}_{\gamma_{0}}^{1 / 2}\left(\gamma_{0}\right) t}, t \in\left[0, T^{*}\right) .
$$


Now we can estimate $C^{\nu}$ norm of $\gamma^{\prime}$. We have

$$
\begin{aligned}
\left\|\gamma^{\prime}(t)\right\|_{C^{\nu}} & \leq\left\|\gamma_{0}^{\prime}\right\|_{C^{\nu}}+\int_{0}^{t}\left\|\nabla u^{\gamma(s)}(\gamma(s)) \gamma^{\prime}(s)\right\|_{C^{\nu}} d s \\
& \leq\left\|\gamma_{0}^{\prime}\right\|_{C^{\nu}}+\int_{0}^{t}\left(\left\|\nabla u^{\gamma(s)}\right\|_{L^{\infty}}\left\|\gamma^{\prime}(s)\right\|_{C^{\nu}}+\left\|\gamma^{\prime}(s)\right\|_{L^{\infty}}\left\|\nabla u^{\gamma(s)}(\gamma(s))\right\|_{C^{\nu}}\right) d s \\
& \leq\left\|\gamma_{0}^{\prime}\right\|_{C^{\nu}}+\int_{0}^{t}\left(\left\|\nabla u^{\gamma(s)}\right\|_{L^{\infty}}\left\|\gamma^{\prime}(s)\right\|_{C^{\nu}}+\left\|\gamma^{\prime}(s)\right\|_{L^{\infty}}\left\|\nabla^{2} u^{\gamma(s)}\right\|_{L^{\infty}}\|\gamma(s)\|_{C^{\nu}}\right) d s \\
& \leq\left\|\gamma_{0}^{\prime}\right\|_{C^{\nu}} \\
& +\int_{0}^{t}\left(C \mathcal{H}_{\gamma_{0}}^{1 / 2}\left(\gamma_{0}\right)\left(\left\|\gamma^{\prime}(s)\right\|_{C^{\nu}}+\left\|\gamma_{0}^{\prime}\right\|_{L^{\infty}}\left\|\gamma_{0}\right\|_{C^{\nu}} e^{C \mathcal{H}_{\gamma_{0}}^{1 / 2}\left(\gamma_{0}\right) s}\right)\right) d s, \quad t \in\left[0, T^{*}\right),
\end{aligned}
$$

where last inequality follows from Lemmata 3.9 and 3.8. Then by the Gronwall Lemma we get the third estimate

$$
\left\|\gamma^{\prime}(t)\right\|_{C^{\nu}} \leq\left(\left\|\gamma_{0}^{\prime}\right\|_{C^{\nu}}+\left\|\gamma_{0}^{\prime}\right\|_{L^{\infty}}\left\|\gamma_{0}\right\|_{C^{\nu}}\right) e^{C \mathcal{H}_{\gamma_{0}}^{1 / 2}\left(\gamma_{0}\right) t}, \quad t \in\left[0, T^{*}\right) .
$$

It remains to find an estimate for $\left\|R^{\gamma(t)}\right\|_{2 \nu}$. We have

$$
R^{\gamma(t)}=R^{\gamma_{0}}+\int_{0}^{t} R^{u^{\gamma(s)}(\gamma(s))} d s, \quad t \in\left[0, T^{*}\right) .
$$

By identity (2.6) we have for $s \in\left[0, T^{*}\right)$

$$
\begin{aligned}
R^{u^{\gamma(s)}(\gamma(s))}(\xi, \eta) & =\nabla u^{\gamma(s)}(\gamma(s, \xi)) R^{\gamma(s)}(\xi, \eta)+\sum_{k}\left(\gamma^{k}(s, \eta)-\gamma^{k}(s, \xi)\right) \times \\
& \int_{0}^{1}\left[\frac{\partial u^{\gamma(s)}}{\partial x_{k}}(\gamma(s, \xi)+r(\gamma(s, \eta)-\gamma(s, \xi)))-\frac{\partial u^{\gamma(s)}}{\partial x_{k}}(\gamma(s, \xi))\right] d r .
\end{aligned}
$$

Therefore,

$$
\left\|R^{u^{\gamma(s)}(\gamma(s))}\right\|_{C_{2}^{2 \nu}} \leq\left\|\nabla u^{\gamma(s)}\right\|_{L^{\infty}}\left\|R^{\gamma(s)}\right\|_{C_{2}^{2 \nu}}+\frac{1}{2}\|\gamma(s)\|_{C^{\nu}}^{2}\left\|\nabla^{2} u^{\gamma(s)}\right\|_{L^{\infty}}, \quad s \in\left[0, T^{*}\right) .
$$

Thus, by inequalities (3.38) and (3.33) we have for $t \in\left[0, T^{*}\right)$

$$
\begin{aligned}
\left\|R^{\gamma(t)}\right\|_{C_{2}^{2 \nu}} & \leq\left\|R^{\gamma_{0}}\right\|_{C_{2}^{2 \nu}}+\int_{0}^{t}\left(\left\|\nabla u^{\gamma(s)}\right\|_{L^{\infty}}\left\|R^{\gamma(s)}\right\|_{C_{2}^{2 \nu}}+\frac{1}{2}\|\gamma(s)\|_{C^{\nu}}^{2}\left\|\nabla^{2} u^{\gamma(s)}\right\|_{L^{\infty}}\right) d s \\
& \leq\left\|R^{\gamma_{0}}\right\|_{C_{2}^{2 \nu}}+\int_{0}^{t}\left(\left\|\nabla u^{\gamma(s)}\right\|_{L^{\infty}}\left\|R^{\gamma(s)}\right\|_{C_{2}^{2 \nu}}+\left\|\gamma_{0}\right\|_{C^{\nu}} e^{C \mathcal{H}_{\gamma_{0}}^{\frac{1}{2}}\left(\gamma_{0}\right) t}\left\|\nabla^{2} u^{\gamma(s)}\right\|_{L^{\infty}}\right) d s \\
& \leq\left\|R^{\gamma_{0}}\right\|_{C_{2}^{2 \nu}}+C\left(\left\|\gamma_{0}\right\|_{C^{\nu}}, \mathcal{H}_{\gamma_{0}}^{1 / 2}\left(\gamma_{0}\right)\right) e^{C \mathcal{H}_{\gamma_{0}}^{1 / 2}\left(\gamma_{0}\right) t} \\
& +\int_{0}^{t} C \mathcal{H}_{\gamma_{0}}^{1 / 2}\left(\gamma_{0}\right)\left\|R^{\gamma(s)}\right\|_{C_{2}^{2 \nu}} d s
\end{aligned}
$$


where in the last inequality we used Lemmata 3.9 and 3.8. Hence, by the Gronwall Lemma we get

$$
\left.\left\|R^{\gamma(t)}\right\|_{C_{2}^{2 \nu}} \leq\left(\left\|R^{\gamma_{0}}\right\|_{C_{2}^{2 \nu}}+C\left(\left\|\gamma_{0}\right\|_{C^{\nu}}, \mathcal{H}_{\gamma_{0}}^{1 / 2}\left(\gamma_{0}\right)\right) e^{C \mathcal{H}_{\gamma_{0}}^{1 / 2}\left(\gamma_{0}\right) t}\right)\right) e^{C \mathcal{H}_{\gamma_{0}}^{1 / 2}\left(\gamma_{0}\right) t}, \quad t \in\left[0, T^{*}\right),
$$

and combining estimates (3.28), (3.31), (3.35), and (3.40) we prove following a-priori estimate

$$
\|\gamma(t)\|_{\mathcal{D}_{\gamma_{0}}} \leq K\left(1+\mathcal{H}_{\gamma_{0}}^{1 / 2}\left(\gamma_{0}\right)\right)\left(1+\left\|\gamma_{0}\right\|_{\mathcal{D}_{\gamma_{0}}}\right)\left\|\gamma_{0}\right\|_{\mathcal{D}_{\gamma_{0}}} e^{C \mathcal{H}_{\gamma_{0}}^{1 / 2}\left(\gamma_{0}\right) t}, \quad t \in\left[0, T^{*}\right),
$$

and the result follows.

\section{REFERENCES}

[1] V. I. Arnold and B. A. Khesin, Topological Methods in Hydrodynamics, Corrected, Springer, 1998.

[2] J. T. Beale, T. Kato, and A. Majda, Remarks on the breakdown of smooth solutions for the 3-D Euler equations, Comm. Math. Phys. 94 (1984), no. 1, 61-66. MR763762 (85j:35154)

[3] J. B. Bell and D. L. Marcus, Vorticity intensification and transition to turbulence in the three-dimensional Euler equations, Comm. Math. Phys. 147 (1992), no. 2, 371-394. MR1174419 (93c:76048)

[4] L. C. Berselli and H. Bessaih, Some results for the line vortex equation, Nonlinearity 15 (2002), no. 6, 17291746, DOI 10.1088/0951-7715/15/6/301. MR1938468 (2003m:76028)

[5] L. C. Berselli and M. Gubinelli, On the global evolution of vortex filaments, blobs, and small loops in $3 D$ ideal flows, Comm. Math. Phys. 269 (2007), no. 3, 693-713, DOI 10.1007/s00220-006-0142-x. MR2276358 (2007m:76027)

[6] H. Bessaih, M. Gubinelli, and F. Russo, The evolution of a random vortex filament, Ann. Probab. 33 (2005), no. 5, 1825-1855, DOI 10.1214/009117905000000323. MR2165581 (2006i:60069)

[7] J.-L. Brylinski, Loop spaces, characteristic classes and geometric quantization, Progress in Mathematics, vol. 107, Birkhäuser Boston Inc., Boston, MA, 1993. MR1197353 (94b:57030)

[8] A. J. Chorin, Vorticity and turbulence, Applied Mathematical Sciences, vol. 103, Springer-Verlag, New York, 1994. MR1281384 (95m:76043)

[9] P. K. Friz, Continuity of the Itô-map for Hölder rough paths with applications to the support theorem in Hölder norm, Probability and partial differential equations in modern applied mathematics, IMA Vol. Math. Appl., vol. 140, Springer, New York, 2005, pp. 117-135, DOI 10.1007/978-0-387-29371-4_8. MR2202036 (2007f:60070)

[10] P. K. Friz and N. B. Victoir, Multidimensional stochastic processes as rough paths, Cambridge Studies in Advanced Mathematics, vol. 120, Cambridge University Press, Cambridge, 2010. Theory and applications. MR2604669 (2012e:60001)

[11] G. Gallavotti, Foundations of fluid dynamics, Texts and Monographs in Physics, Springer-Verlag, Berlin, 2002. Translated from the Italian. MR1872661 (2003e:76002)

[12] M. Gubinelli, Controlling rough paths, J. Funct. Anal. 216 (2004), no. 1, 86-140, DOI 10.1016/j.jfa.2004.01.002. MR2091358 (2005k:60169)

[13] M. Hairer, Rough stochastic PDEs, Comm. Pure Appl. Math. 64 (2011), no. 11, 1547-1585, DOI 10.1002/cpa.20383. MR2832168

[14] Solving the KPZ equation, Ann. Math. (2013). to appear.

[15] H. Helmholtz, Über Integrale der hydrodynamischen Gleichungen, welche den Wirbelbewegungen entsprechen., Journal für die reine und angewandte Mathematik (Crelles Journal) 1858 (1858), no. 55, 25-55, DOI 10.1515/crll.1858.55.25.

[16] W. Thomson (Lord Kelvin), On vortex motion, Trans. Royal Soc. Edin. 25 (1869), 217-260.

[17] T. J. Lyons, Differential equations driven by rough signals, Rev. Mat. Iberoamericana 14 (1998), no. 2, 215-310, DOI 10.4171/RMI/240. MR1654527 (2000c:60089)

[18] T. J. Lyons, M. Caruana, and T. Lévy, Differential equations driven by rough paths, Lecture Notes in Mathematics, vol. 1908, Springer, Berlin, 2007. Lectures from the 34th Summer School on Probability Theory held in Saint-Flour, July 6-24, 2004; With an introduction concerning the Summer School by Jean Picard. MR2314753 (2009c:60156) 
[19] T. Lyons and Z. Qian, System control and rough paths, Oxford Mathematical Monographs, Oxford University Press, Oxford, 2002. Oxford Science Publications. MR2036784 (2005f:93001)

[20] L. Rosenhead, The Spread of Vorticity in the Wake Behind a Cylinder, Proceedings of the Royal Society A: Mathematical, Physical and Engineering Sciences 127 (1930), no. 806, 590-612, DOI 10.1098/rspa.1930.0078.

[21] A. Vincent and M. Meneguzzi, The Spatial Structure and Statistical Properties of Homogeneous Turbulence, Journal of Fluid Mechanics 225 (1991), 1-20, DOI 10.1017/S0022112091001957.

[22] L. C. Young, An inequality of the Hölder type, connected with Stieltjes integration, Acta Math. 67 (1936), no. 1, 251-282, DOI 10.1007/BF02401743. MR1555421

${ }^{\dagger}$ Department of Mathematics, University of York, Heslington, UK, ${ }^{\ddagger}$ CEREMADE \& CNRS UMR 7534, UnIVERSITÉ PARIS-DAUPHINE, FRANCE 